\title{
TOMATO SECONDARY TRISOMICS: ORIGIN, IDENTIFICATION, MORPHOLOGY, AND USE IN CYTOGENETIC ANALYSIS OF THE GENOME
}

\author{
GURDEV S. KHUSH \\ International Rice Institute, Manila Hotel, Manila, Philippines \\ and \\ CHARLES M. RICK \\ Department of Vegetable Crops, University of California, \\ Davis, California, U.S.A. 95616
}

Received 27.iii.68

\section{INTRODUCTION}

SECONDARY trisomics, i.e. individuals having an isochromosome in addition to the normal somatic number, have been least investigated among the known kinds of trisomics. The lack of any cytogenetic technique for the direct experimental production of secondary trisomics probably accounts for the rarity of studies on this subject. Definitely proven cases of secondary trisomy have been reported only in Datura, maize, tomato, and wheat. In Datura stramonium, 14 of the 24 possible secondary trisomics were established (Blakeslee and Avery, 1938). Only one secondary trisomic of maize (Rhoades, 1933, 1940) and one of wheat (Sears, 1954) and two of tomato (Sen, 1952; Moens, 1965) have been reported to date.

The identification of secondary trisomics was confirmed in Datura stramonium by extensive breeding tests and in maize and tomato by the highly diagnostic pachytene analysis. Despite their utility for finding the arm location of the markers, thus ascertaining the position of the centromeres and the orientation of linkage maps, secondary trisomics have not been employed heretofore for such analysis in any species.

In recent work, we have isolated nine secondary trisomics of tomato including one reported by Sen (1952). Five of these were employed in determining the arm location of marker genes. Knowledge of the tomato genome as well as understanding of secondary trisomy in general has been considerably enhanced by these investigations on morphology, genetics, cytology and transmission rates.

\section{ORIGIN}

Sen (1952) working with tomato and Sears (1954) with wheat were the first to isolate secondary trisomics amongst the progenies of plants in which a normal chromosome of the complement had been replaced by an isochromosome for one of its arms. Such individuals which are haploid for one arm and triploid for the other arm of the same chromosome were called monoisosomes by Sears (1954) and haplo-triplo-disomis (HTD's) by Khush and Rick (1967b). We were able to establish seven HTD's in tomato, from five of which large progenies were raised, three yielding secondary trisomics. A procedure for obtaining haplo-triplo-disomics, thereby providing a source of secondary trisomics, was also outlined by Khush and Rick (1967b). Three 
secondary trisomics-2n+8L $-8 \mathrm{~L}, 2 \mathrm{n}+9 \mathrm{~L} \cdot 9 \mathrm{~L}$ and $2 \mathrm{n}+10 \mathrm{~L} \cdot 10 \mathrm{~L}$-were thus produced experimentally.

Three secondary trisomics were isolated from the progenies of double iso-compensating trisomics. In such trisomics, one normal chromosome of the complement is missing, but it is compensated by two isochromosomes, one for each of the two arms. Two compensating trisomics of this kind$2 \mathrm{n}-7 \mathrm{~S} \cdot 7 \mathrm{~L}+7 \mathrm{~S} \cdot 7 \mathrm{~S}+7 \mathrm{~L} \cdot 7 \mathrm{~L}$ and $2 \mathrm{n}-3 \mathrm{~S} \cdot 3 \mathrm{~L}+3 \mathrm{~S} \cdot 3 \mathrm{~S}+3 \mathrm{~L} \cdot 3 \mathrm{~L}$ - have been obtained in tomato (Khush and Rick, 1967a). In the same publication we predicted that $2 n-3 S \cdot 3 L+3 S \cdot 3 S+3 L \cdot 3 L$ should yield $2 n+3 S \cdot 3 S$ in its progeny but not $2 n+3 L \cdot 3 L$. The unbalance caused by the extra $3 \mathrm{~L} \cdot 3 \mathrm{~L}$ is not tolerated by the gametophytic generation (Khush and Rick, 1967b). Similarly, the appearance of $2 n+7 S \cdot 7 S$ and $2 n+7 L \cdot 7 L$ in the progeny of $2 \mathrm{n}-7 \mathrm{~S} \cdot 7 \mathrm{~L}+7 \mathrm{~S} \cdot 7 \mathrm{~S}+7 \mathrm{~L} \cdot 7 \mathrm{~L}$ was also predicted. The data on the backcross progenies of these two trisomics are presented in table 1. As predicted, both

TABLE 1

Backcross progenies of double-iso compensating trisomics

\begin{tabular}{|c|c|c|c|c|c|c|}
\hline & & & & $\underbrace{\text { ogeny }}$ & & \\
\hline & & $\overbrace{}^{2 n}$ & $\begin{array}{c}\text { Parental } \\
2 n+1\end{array}$ & $\begin{array}{c}\text { Secondary } \\
\text { trisomic } \\
\text { for short } \\
\text { arm }\end{array}$ & $\begin{array}{l}\text { Secondary } \\
\text { trisomic } \\
\text { for long } \\
\text { arm }\end{array}$ & \\
\hline Trisomic & Total & No. $\%$ & No. $\%$ & No. \% & No. $\%$ & Other \\
\hline $2 n-3 S \cdot 3 L+3 S \cdot 3 S+3 L \cdot 3 L$ & 283 & $195 \quad 68 \cdot 8$ & $68 \quad 24 \cdot 0$ & $5 \cdot 6$ & $0 \cdot 0$ & $4 *$ \\
\hline $2 \mathrm{n}-7 \mathrm{~S} \cdot 7 \mathrm{~L}+7 \mathrm{~S} \cdot 7 \mathrm{~S}+7 \mathrm{~L} \cdot 7 \mathrm{~L}$ & 185 & $116 \quad 62 \cdot 7$ & $48 \quad 25 \cdot 9$ & $20 \quad 10 \cdot 8$ & $0 \cdot 54$ & 0 \\
\hline
\end{tabular}

of the compensating trisomics yielded secondary trisomics for short arms among their progenies at a relatively high frequency (table 1, columns 7 and 8) ; no secondary trisomic for $3 \mathrm{~L}$ appeared, but one individual of $2 n+7 \mathrm{~L} \cdot \mathrm{L} 7$ constitution was produced. On the basis of cytological observations, a much higher frequency of $2 n+7 \mathrm{~L} \cdot 7 \mathrm{~L}$ individuals would be expected, but the poor viability of the $\mathrm{n}+7 \mathrm{~L} \cdot 7 \mathrm{~L}$ gametophytes is probably responsible for low yield obtained. Three plants in the progeny of $2 n-3 S \cdot 3 L+3 S \cdot 3 S+3 L \cdot 3 L$ were HTD's $(2 n-3 S \cdot 3 L+3 L \cdot 3 L)$. The argument that $n-3 S \cdot 3 L+3 L \cdot 3 L$ gametes are viable might seem to be favoured by the appearance of these HTD's but not by the failure of the earlier tested $2 n-3 S \cdot 3 L+3 L \cdot 3 L$ to transmit its karyotype to a large progeny (Khush and Rick, 1967b). Therefore, these three HTD's must have originated by somatic loss of the $3 \mathrm{~S} \cdot 3 \mathrm{~S}$ chromosome during early ontogeny from offspring that initially were $2 n-3 S \cdot 3 L+3 S \cdot 3 S+3 L \cdot 3 L$. This explanation seems plausible also in view of the fact that the telocentric $\cdot 3 \mathrm{~S}$ from which the $3 \mathrm{~S} \cdot 3 \mathrm{~S}$ isochromosome originated was also somatically unstable (Khush and Rick, 1967a).

Of the other three secondary trisomics, $2 n+9 S \cdot 9 S$ and $2 n+6 L \cdot 6 L$ originated spontaneously in the progenies of haplo-6S $\cdot 7 \mathrm{~S}$ and haplo-4S $10 \mathrm{~S}$, respectively (Khush and Rick, 1966a). The remaining one-2n+12L $-12 \mathrm{~L}$ -is the only secondary trisomic which originated on three independent occasions. It appeared spontaneously, first in the progeny of a plant deficient 
for 4S (Khush and Rick, 1967c) and second in the progeny of haplo 3Striplo 3L. Finally, it was isolated among the progeny of haplo-10S $\cdot 12 \mathrm{~S}$. In the latter family, the isochromosome $12 \mathrm{~L} \cdot 12 \mathrm{~L}$ probably resulted from the fracture of the tertiary chromosome $10 \mathrm{~L} \cdot 12 \mathrm{~L}$ in the centromere region, and its inclusion in a gamete carrying $n$ chromosomes led to the production of said trisomic.

Thus, in summary, our set of nine secondary trisomics appeared among the progenies of haplo-triplo-disomics, double iso-compensating trisomics, tertiary monosomics and segmental deficiencies. The mechanism that leads to the production of secondary trisomics seems clear. First, an isochromosome is produced by breakage of the centromere and union of identical arms. Second, the isochromosome must be included in a gametophyte in addition to the entire haploid chromosome complement, and such a gametophyte must be viable. The isochromosomes $2 \mathrm{~L} \cdot 2 \mathrm{~L}, 3 \mathrm{~L} \cdot 3 \mathrm{~L}$ and $6 \mathrm{~L} \cdot 6 \mathrm{~L}$ produce so much unbalance as extras that the gametophytes fail to function-a topic that is treated in detail in a subsequent section. Third, the $\mathrm{n}+\mathrm{isochromo-}$ some gamete must be fertilised by an $\mathrm{n}$ gamete and the resulting zygote must be viable.

Until recently, no way of producing isochromosomes experimentally was known (Avery, Satina and Rietsema, 1959, p. 101). We have shown, however, (Khush and Rick, 1967b) that isochromosomes can be produced by irradiating pollen. The hypothesis of Belling and Blakeslee (1924) that isochromosomes arise by crossing over between sister strands that lie side by side, but with the ends of one reversed, seems less probable.

\section{Gytology}

Familiarity with the gross morphology of primary trisomics is often helpful in identifying new trisomic types, but may be untrustworthy as the sole criterion according to the present research; conclusive evidence is provided by cytological examination. The secondary trisomic condition can be readily diagnosed by the presence of ring trivalents. Pachytene configurations (figs. 1-18) are then easily analysed to identify the isochromosome, which may be present as a univalent when paired internally (figs. 1, 4, $7,8,9,12,14$ and 15). Chromomere-by-chromomere pairing is clearly evident and terminal centromeres can be seen in all of these figures. Frequently the isochromosome is paired with the two normal homologues to form a trivalent (figs. 2, 3, 5, 6, 10, 11, 13, 16, 17 and 18). When univalent, the isochromosome forms a small ringlet. Evidently, internally paired isochromosomes invariably form at least one chiasma. Cells with three univalents were extremely rare. The frequency of cells with trivalents was highly variable (table 2). In general, secondary trisomics for long arms formed trivalents more frequently than those for short arms, as expected on the basis of opportunity for pairing and chiasma formation.

The trivalents assumed various configurations. The eight different types observed and their frequencies in each of the trisomics are shown in table 2. These different configurations result from a variable number and position of chiasmata. The pachytene associations and the number and position of chiasmata that give rise to these configurations at diakinesis are shown diagramatically in fig. 20. It may be noted that the frequency of configurations of type $g$ was lowest. In fact, we observed only two PMC's 


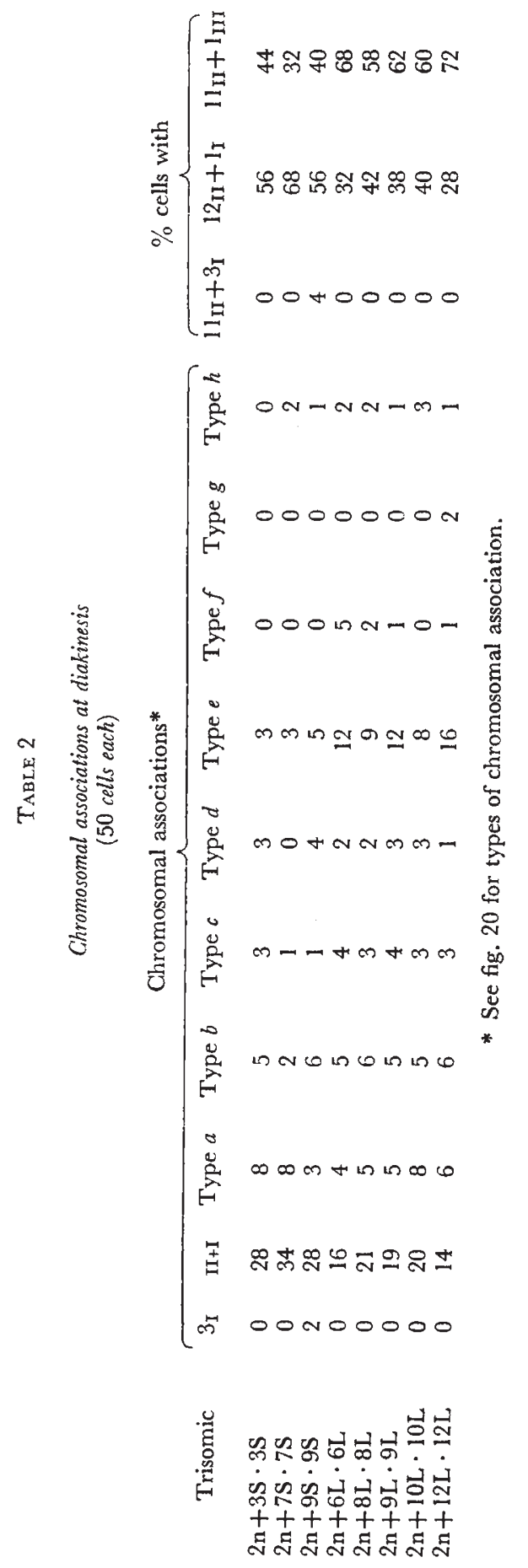



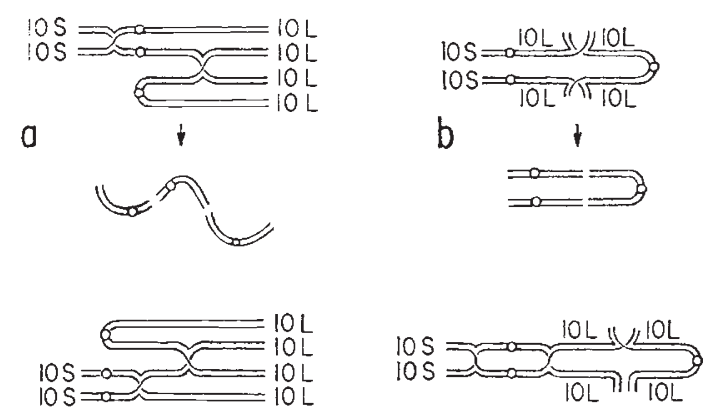

$c$
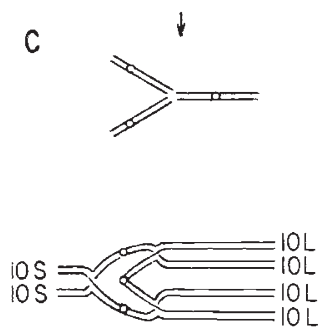

e
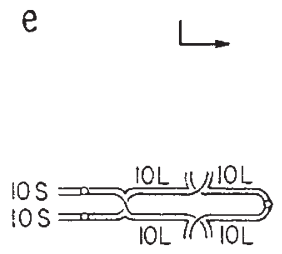

f
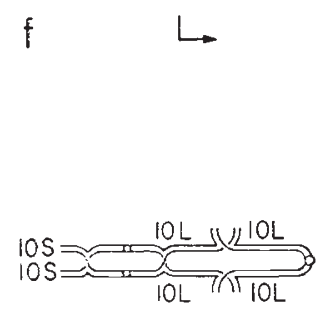

$\mathrm{g}$
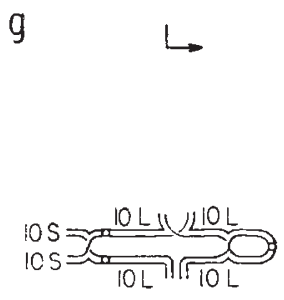

h

Frg. 20.-Patterns of chromosomal association and chiasma formation at pachytene in the trivalent of the secondary trisomic $2 \mathrm{n}+10 \mathrm{~L} \cdot 10 \mathrm{~L}$. Arrows point to the respective configurations anticipated at diakinesis. 
with trivalents of this type, both in $2 \mathrm{n}+12 \mathrm{~L} \cdot 12 \mathrm{~L}$. The fact that such a trivalent requires the highest number of chiasmata probably explains its rarity. The trivalents of types $f$ and $h$ were also infrequent, ring and chain trivalents (types $a$ to $e$ ) being more common. Rings were more frequent in the secondaries for long arms than in those for short arms.

The later stages of meiosis were normal. When present as a univalent the isochromosome passed to one of the poles at anaphase I, laggards having appeared rarely. The orientation of the trivalent at metaphase I and the subsequent segregation of the three chromosomes at anaphase I deserve special mention. In the secondary trisomics for such long arms as $6 \mathrm{~L}, 8 \mathrm{~L}$, $9 \mathrm{~L}$ and $10 \mathrm{~L}$, the orientation of the trivalent frequently favoured the separation of the isochromosome to one pole and the two normal chromosomes to the other, probably for mechanical reasons. Consisting of two long arms, the isochromosome is much longer than the entire normal chromosome and tends to orient itself toward one pole, the two normal chromosomes toward the other. An example may be seen in the ring trivalent of $2 n+10 \mathrm{~L} \cdot 10 \mathrm{~L}$ shown in fig. 19. In trivalents of the secondary trisomics for short arms, such as $3 \mathrm{~S}, 7 \mathrm{~S}$ and $9 \mathrm{~S}$, the reverse is true. The isochromosome in these trisomics is shorter than the two normal chromosomes, and the orientation of the trivalents tends to result in the two normal chromosomes passing to different poles and the isochromosome segregating at random. Since the centromere of chromosome 12 is median, the three members of the trivalent in $2 \mathrm{n}+12 \mathrm{~L} \cdot 12 \mathrm{~L}$ are identical in size, the isochromosome could not be distinguished, consequently the orientation and segregation of the members of the trivalent could not be determined. Because of their similar size, the orientation and segregation was probably random.

This variable behaviour of the trivalents of different secondary trisomics at metaphase-anaphase I has an obvious bearing on the gametic output. Four kinds of gametes can be produced by any secondary trisomic by two types of segregations of three chromosomes of the trivalent. For example, from a trivalent $10 \mathrm{~S} \cdot 10 \mathrm{~L}-10 \cdot \mathrm{L} 10 \mathrm{~L}-10 \mathrm{~L} \cdot 10 \mathrm{~S}$ of $2 \mathrm{n}+10 \mathrm{~L} \cdot 10 \mathrm{~L}$, the $10 \mathrm{~S} \cdot 10 \mathrm{~L}$ and $10 \mathrm{~L} \cdot 10 \mathrm{~L}$ chromosomes may go to one pole and $10 \mathrm{~S} \cdot 10 \mathrm{~L}$ to the other. This type of segregation should produce $\mathrm{n}$ and $\mathrm{n}+10 \mathrm{~L} \cdot 10 \mathrm{~L}$ gametes. The segregation in which the $10 \mathrm{~L} \cdot 10 \mathrm{~L}$ chromosome goes to one pole and the two $10 \mathrm{~S} \cdot 10 \mathrm{~L}$ chromosomes to the other should produce $\mathrm{n}-10 \mathrm{~S} \cdot 10 \mathrm{~L}+10 \mathrm{~L} \cdot 10 \mathrm{~L}$ and $n+10 S \cdot 10 \mathrm{~L}$ gametes. The gametes of the former type $(\mathrm{n}-10 \mathrm{~S} \cdot 10 \mathrm{~L}+$ $10 \mathrm{~L} \cdot 10 \mathrm{~L})$ must certainly abort due to the deficiency of one arm $(10 \mathrm{~S})$. Had such gametes been viable they would have produced haplo-triplo-disomics. As already shown by Khush and Rick (1967b), such gametes never function. Therefore, the three types of functional gametes produced by a secondary trisomic such as $2 \mathrm{n}+10 \mathrm{~L} \cdot 10 \mathrm{~L}$ are $\mathrm{n}, \mathrm{n}+10 \mathrm{~L} \cdot 10 \mathrm{~L}$ and $\mathrm{n}+10 \mathrm{~S} \cdot 10 \mathrm{~L}$, producing diploids, secondary trisomics and the related primary trisomics, respectively, upon backcrossing.

The appearance of related primary trisomics in the progenies of the secondary trisomic is one of the very important features of these trisomicsa subject that will be treated in later sections. It should be emphasised here, however, that the frequency of $n+l$ gametes in which the extra chromosome is primary would depend upon the frequency of trivalent formation and the type of segregation it undergoes at anaphase I. The extra isochromosomes for the short arms form trivalents less frequently, which, when formed, mainly segregate to produce $n$ and $\mathrm{n}+1$ gametes in which the extra chromosome is 
the isochromosome. With the secondary trisomics for long arms, the cytological conditions for production of $n+1$ gametes with a primary extra chromosome are more favourable.

\section{Morphology}

The secondary trisomics of tomato differ to varying degrees from diploid, in vigour and growth habit. The secondaries for the long arms, 6L, 7L, 8Ls $9 \mathrm{~L}$ and $10 \mathrm{~L}$, have much slower growth rates at all stages of growth than those for the short arms. Moreover, the secondaries for the long arms are sometimes difficult to distinguish from the corresponding primaries. Most of the characters of the primaries tend to be exaggerated in the secondaries, all of the latter being distinguishable from the normal sibs at the cotyledonary or first true leaf stages. On the other hand, the seedling vigour of the secondaries for the short arms such as 3S, 7S and 9S is scarcely reduced and the morphology of their seedlings is not modified to any appreciable extent. Thus, as seedlings, $2 n+3 S \cdot 3 S$ and $2 n+9 S \cdot 9 S$ cannot be separated from the diploids and $2 n+7 S \cdot 7 S$ only with difficulty. Under field conditions, $2 n+9 S \cdot 9 S$ is rather weak and strikingly different in growth habit, but $2 n+3 S \cdot 3 S$ and $2 n+7 S \cdot 7 S$ are almost as vigorous as diploids. The remaining secondary, $2 n+12 L \cdot 12 L$, is quite distinct at all stages of growth, but it shows only about half of the characters of its primary.

The following morphological descriptions of the secondary trisomics are based on the observations of both greenhouse and field-grown plants. Observations on $2 n+6 L \cdot 6 L$ and $2 n+7 L \cdot 7 L$ were limited to greenhouse cultures of the single plant of each that have been obtained to date.

$2 n+3 S \cdot 3 S$. Plant vigorous and fruitful. Resembles triplo-3 in being somewhat slender and in having flower buds which taper towards the pedicel, thus sharply demarcating the receptacle; pedicel sharply recurved, up to $180^{\circ}$, appearing like a hook; the inflorescences highly pubescent; terminal meristems resembling the Hrt mutant. The hairless appearance of triplo- 3 must be controlled by $3 \mathrm{~L}$. In respect to hair density, the two arms thus seem to act in opposite directions.

$2 n+6 L \cdot 6 L$. Plant very weak and unfruitful; distinguishing features of triplo-6 exaggerated; all the plant parts including internodes, leaf segments, inflorescences and flowers elongate. The highly elongated internodes of the seedling render it so different from diploid sibs, in fact, that they provoked cytological examination and identification. All parts of the inflorescence are extremely elongate; the characteristic leaf texture of triplo-6 magnified. Like triplo-6, this trisomic is somewhat fragile.

$2 n+7 S \cdot 7 S$. Plant vigorous and fruitful; leaves curled and serrate like those of triplo-7. But in all of the other distinguishing characters this trisomic is the antithesis of triplo-7. Triplo-7, for example, has compact and bushy growth habit, thickened stems, compact internodes and inflorescences, and disc-like fruit, whereas $2 n+7 S \cdot 7 S$ has open growth habit, slender stems and other plant parts, elongated internodes, inflorescences and flowers, and somewhat elongated fruits.

$2 n+7 \mathrm{~L} \cdot 7 \mathrm{~L}$. Plant very slow and weak; most of the characters of triplo-7 exaggerated: for example, stems very stiff and heavy with small internodes; leaves extremely curled with deeper but fewer serrations; tendency 
towards blindness extremely pronounced, every branch losing its growing point after producing 2-3 leaves. Due to the latter defect, the plant produced flower buds only after 10 months of growth and none of its flowers have set fruit yet.

$2 n+8 L \cdot 8 L$. Plant very weak and slow growing, half the size of triplo-8; growth habit open and erect; all other characters of triplo-8 magnified: cotyledons much thicker, broader and leathery; internodes much shorter; leaves entirely convex, greyish, deep veined and thickened with very little serration and with fewer divisions; flower parts, including the pedicels, reduced in size; flower partially cleistogamous, stigma exserted; and fruits flattened and white. Moderately fruitful under greenhouse conditions.

$2 n+9 S \cdot 9 S$. Plant very vigorous in the seedling stage but later growth much retarded; highly unfruitful in the greenhouse and under field conditions. The trisomic shows no resemblance to triplo-9 except for a tendency for blindness. Leaves simple with secondary leaflets less serrate and more rounded; inflorescence, in contrast with the compound structure of triplo-9, simple with very few abortive flowers; flowers with thickened receptacle and long and dialytic anthers; flowers and fruits larger than those of triplo-9.

$2 n+9 L \cdot 9 L$. Plant very diminutive and slow growing, one-third the size of triplo-9. All the characters of triplo-9 are highly exaggerated in this trisomic: growth habit erect; leaves folded, plicate; inflorescence compound; flowers and fruits smaller; stem colour yellow-green; internodes reduced in size and number; plant somewhat brittle; moderately fruitful under greenhouse conditions.

$2 \mathrm{n}+10 \mathrm{~L} \cdot 10 \mathrm{~L}$. Plant slow growing and about half the size of triplo-10. In common with all the secondaries for long arms, all the characters of the corresponding primary (triplo-10) are exaggerated in this trisomic: stem thicker; growth habit stocky and bushy; internodes very short; inflorescence compact; flower parts reduced in size; stigma exserted; undulations of leaf margins extremely pronounced; terminal leaf segment twisted. Unlike triplo-10, flower buds have intense anthocyanin, even in summer, and calyx is longer than corolla.

$2 n+12 L \cdot 12 \mathrm{~L}$. This trisomic resembles triplo- 12 in about half of its characters. Its growth habit is somewhat like triplo-12, but it is not as compact. Leaves are curled but the characteristic fimbriation of the leaves of triplo- 12 is absent. The colour of leaves is deep grey-green instead of the bluish-grey of triplo-12. Leaves are broader, but not so serrate. It differs from triplo- 12 in having an elongate inflorescence, but resembles it in having a reduced number of flowers per inflorescence. The pedicel is elongate. This trisomic has an extreme tendency towards blindness. Almost 90 per cent. of the seedlings go blind after producing one or two leaves. If left undisturbed in the seed pan, about 75 per cent. of them later produce axillary shoots, the remaining 25 per cent. not producing any further growth. This tendency for blindness is evident in the mature plants, as many shoots lose their growing points. Due to this inherent defect, the vegetative growth is one-half to one-third that of 
triplo-12. Some flowers have normal anthers while in others one to five anthers may be degenerated. The anthers show varying degrees of adnation with the ovary. The green and ripe fruits may thus show one to five suture-like markings on the surface.

From the above morphological descriptions of the secondary trisomics, the following conclusions can be drawn:

(a) The long arms of chromosomes with sub-median centromeres exert a disproportionately greater influence than the short arms on the morphology of trisomic plants. The same conclusion was drawn from the study of telotrisomics (Khush and Rick, 1967d) and tertiary trisomics (Khush and Rick, 1967e). All of the morphological features of the related primary trisomic can, in fact, be easily seen in the telotrisomics and secondary trisomics for long arms.

(b) The secondary trisomics for short arms, like short arm telotrisomics, show scarcely any resemblance to the related primary trisomic. Most of the characters by which the secondary trisomics and telotrisomics for short arms differ from the diploids are therefore unpredictable. Since the differences from $2 n$ sibs are small they can be distinguished only with difficulty.

(c) The secondary trisomic for the long arm of the metacentric chromosome 12 shows about half of the characters of the related primary trisomic and in general looks quite different from it. It therefore appears that the two arms of this chromosome contribute about equally to the morphology of the primary trisomic. The same may be true for the other two metacentric chromosomes of the complement, 5 and 11. The secondary trisomic for the short arm of metacentric chromosome 5 of maize (Rhoades, 1933) showed a similar relation to its primary.

(d) A finding of special interest that has emerged from the morphological examination of the secondary trisomics is that some primary trisomic characters result from the interaction of the two arms, which when present alone influence the morphology in opposite directions. Thus, $3 \mathrm{~S}$ acts to produce pubescent stems and inflorescences in contrast to the glabrous stems and inflorescences conditioned by $3 \mathrm{~L}$. Similar relations can be revealed by comparing the morphology of $2 n+7 S \cdot 7 S$ and $2 n+7 L \cdot 7 L$ with that of $2 n+7 S \cdot 7 L$ and the same for $2 n+9 S \cdot 9 L$. Compared with the diploid, $2 n+7 S \cdot 7 L$, for example, has thick stems, broad leaves, short internodes and compact inflorescences, which result in a bushy growth habit. All these characters are magnified in $2 \mathrm{n}+7 \mathrm{~L} \cdot 7 \mathrm{~L}$, which has even thicker stems, broader leaves, shorter internodes, more compact inflorescences and bushier growth habit. In contrast, the morphology of $2 n+7 \mathrm{~S} \cdot 7 \mathrm{~S}$ is modified in the opposite direction. This trisomic has slender stems and leaves, longer internodes and inflorescences, and an open growth habit. Similarly, all the plant parts of $2 n+9 S \cdot 9 \mathrm{~L}$ including internodes, leaves, flowers and fruits are somewhat smaller than the diploid, and in $2 n+9 L \cdot 9 L$ they are even smaller. However, in $2 n+9 S \cdot 9 S$ all these organs are bigger than in the diploid. It is therefore evident that the primary trisomics are intermediate between the two related secondaries in several characters. A similar relationship was found for several primary and secondary trisomics of Datura stramonium by Blakeslee and his associates (Avery, Satina and Rietsema, 1959, p. 89). 


\section{Genetics}

The genetic ratios are modified in the same fashion in the progenies of secondary trisomics as in the progenies of telotrisomics (Khush and Rick, $1967 d$ ) and tertiary trisomics (Khush and Rick, 1966b, 1967e). The nature of the modified ratios permits the determination of arm locations of markers. All the gametophytes that receive an isochromosome in place of a normal chromosome abort because one arm is deficient; consequently, the $n$ gametes and resultant $2 \mathrm{n}$ zygotes produced by secondaries can only consist of normal homologues. Therefore, if a secondary trisomic carries a recessive marker on one normal chromosome and the dominant allele on the normal homologue, the diploid fraction of its $\mathrm{F}_{2}$ and $\mathrm{BC}$ progeny should segregate $3: 1$ and $1: 1$, respectively. All of the secondary trisomic progeny should have the normal phenotype if the marker has its locus on the isochromosome. If the marker is not located on the duplicated arm, normal disomic ratios should obtain for the secondary trisomic fraction. Observed ratios, whether $3: 1::$ all:0 or $3: 1:: 3: 1$ for the $\mathrm{F}_{2}$; or $1: 1::$ all:0 or $1: 1:: 1: 1$, in the $\mathrm{BC}$, can thereby identify the appropriate arm for the marker. In the telotrisomic and tertiary trisomic progenies giving trisomic segregations, an occasional trisomic individual with recessive phenotype may be obtained by double reduction. Such individuals were in fact obtained in the progenies of several tertiary trisomics and telotrisomics. However, if a secondary trisomic progeny segregates in a trisomic fashion, no secondary trisomic with recessive phenotype should appear, as only one arm, but not both, of the isochromosome can receive the recessive allele by crossing over. Thus, as soon as a secondary trisomic with recessive phenotype is observed, it can be safely concluded that the progeny is segregating in disomic fashion and that no further scoring is necessary.

As will be discussed in the next section, the progenies of most of the secondary trisomics regularly include related primary trisomics, all of which should have normal phenotype if the marker is situated very near the centromere. If the marker is located away from the centromere, however, an occasional primary trisomic with the recessive phenotype may be obtained. Thus, if an appreciable number of primary trisomics appear in the segregating secondary trisomic progeny, approximate location of the marker in relation to the centromere can be determined. Moreover, by studying the genetic ratios among the primary trisomic fraction of the progeny, chromosomal location of the marker can be verified.

From the foregoing discussion, it is evident that three kinds of ratios may be obtained among the progenies of most of the secondary trisomics. The ratio among the diploid fraction should vary from disomic for genes near the centromere to lower values for those more distally located. For a heterozygote with a recessive gene on one of the normal chromosomes and the dominant allele in the three other homologous arms, the minimal gametic ratio expected of a locus enjoying free recombination between the four arms would be $3: 1$. Among the primary trisomic fraction, the ratio should be trisomic if the marker is located on that chromosome. Finally, the secondary trisomic fraction should give trisomic ratios if the marker is located on the isochromosome arm and disomic ratios if it is located on the other arm. Therefore, those secondary trisomics which yield an appreciable number of primary trisomics in their progenies can be utilised, like compensating 
trisomics (Khush and Rick, 1967a), for simultaneous assignment of unlocated markers to their respective chromosomes and arms.

According to Avery, Satina and Rietsema (1959), the secondary trisomics of Datura stramonium were utilised for determining the arm location of several markers. As far as we are aware, however, no published data on the secondary trisomic ratios obtained in Datura stramonium or any other species are available. Blakeslee (1924) presented data on the segregating progenies of two secondary and the two related primary trisomics. Both of the primary trisomics gave trisomic ratios with their respective genes, but, according to the author, only one secondary gave trisomic ratios, not the other. These data are unsatisfactory for the following reasons.

Firstly, one of the two supposed secondaries, wiry, was later found to be a tertiary trisomic. Secondly, the cytological nature of the secondary trisomic at that time was not clear, and Blakeslee supposed that the ratios for the primary and secondary trisomics should be similar-a supposition that we now know for reasons outlined above to be erroneous. Thirdly, for the sake of " convenience", he presented data on the diploid fraction of the progenies only. We know now that ratios in the diploid portions of the progenies of secondary and tertiary trisomics should remain unmodified. These data are therefore useless. Moreover, he obtained an 8:1 ratio for $p$ in the diploid portion of progeny of " poinsettia " (a primary trisomic) as well as in the diploid portion of the progeny of "wiry" (a tertiary trisomic). Such a ratio is expected for the former trisomic, but not for the latter. Similarly, he obtained a ratio of $8: 1$ for $a$ in the diploid portion of "cocklebur" (a primary) and a ratio of $3: 1$ for the same gene in the diploid fraction of "wedge" (a related secondary). The trisomic nature of the former segregation is unquestionable, but his interpretation of the diploid nature of the latter is invalid. All of the secondary trisomics of the progeny may have had normal phenotype with respect to $a$, and the ratio may have been trisomic.

In view of the above considerations, the data from the segregating progenies of secondary trisomics of tomato can be analysed to yield information about the chromosomal and arm location of the markers and their approximate distances from the centromere. Five secondary trisomics were used to study segregation of 10 genes belonging to four chromosomes of the complement (table 3 ). Of the remaining four secondaries, $2 \mathrm{n}+6 \mathrm{~L} \cdot 6 \mathrm{~L}$ does not transmit, thus $F_{1}$ trisomics could not be obtained, and the other three were obtained so recently that they have not yet been employed in genetic studies. As may be seen from table 3, the diploid portions of all of the progenies gave, as expected, essentially disomic ratios. Among the primary trisomic fractions all the markers except $l g$ gave trisomic ratios. Since $l g$ segregated in diploid fashion among the primary trisomic fraction, the previous assignment of this marker by linkage test (Butler, 1954) to chromosome 10 must have been in error. Three triplo-10's with ag phenotype were produced by double reduction, indicating thereby that ag must be distant from the centromere. In the same family, all the triplo-10's were $h^{+}$, thus suggesting that $h$ is closer to the centromere than ag. Similarly, since one triplo-12 with alb phenotype, but none with $f d$, was obtained, it appears that alb is more distal from the centromere of 12 than $f d$. However, the number of primary trisomics in both of the families was so small that the foregoing conclusion must at best be considered tentative. The data of table 3 show that all the genes except $a g$ and $h$ segregated in disomic fashion among the secondary trisomic 


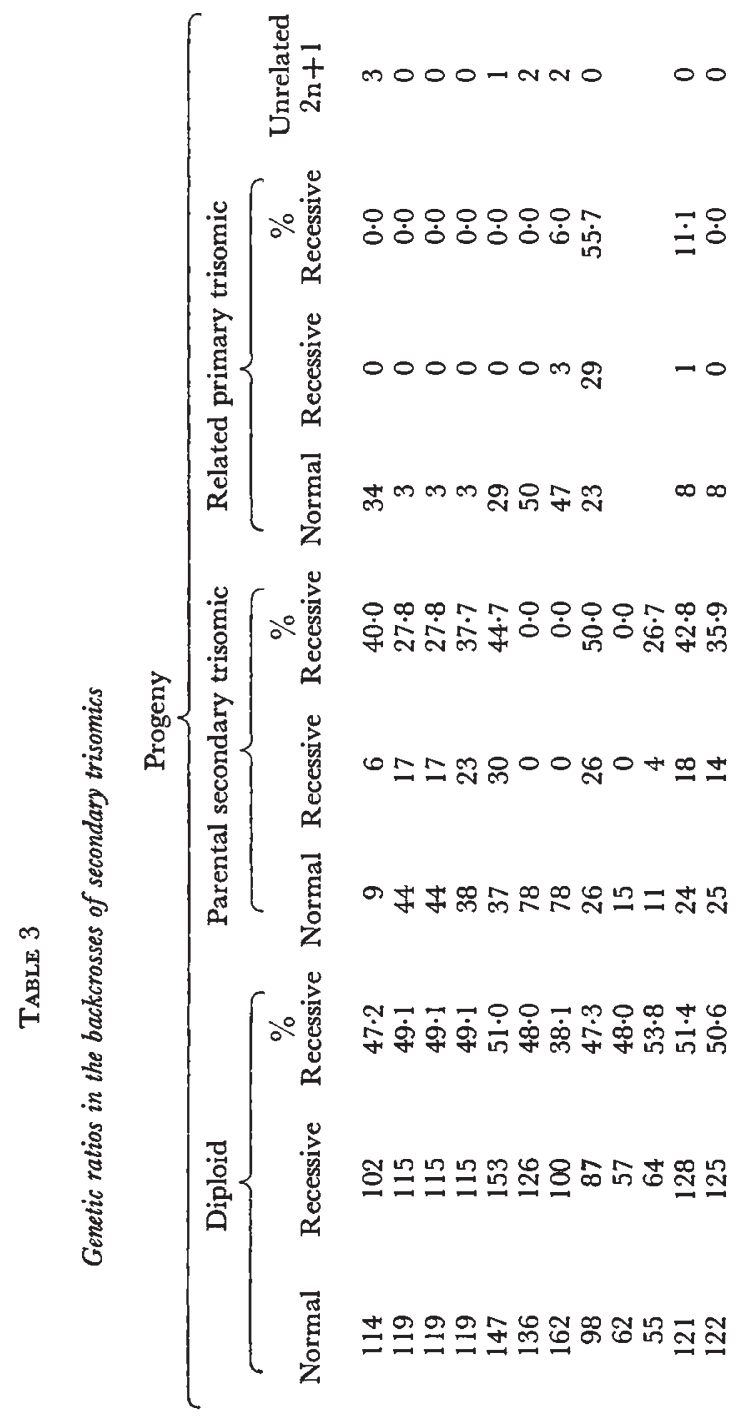

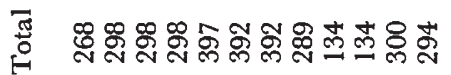

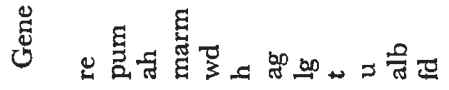

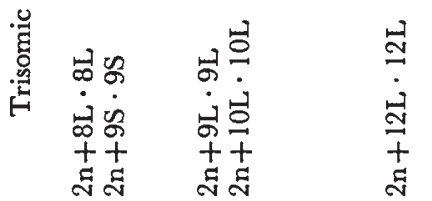


fractions. Probably because of reduced viability, the number of secondary trisomics with recessive phenotypes was less than 50 per cent. in most of the families giving disomic ratios. But there was hardly any doubt about the disomic inheritance in these families. All of the secondary trisomics of the family segregating for $a g$ and $h$ had normal phenotype for these genes, and the trisomic nature of this segregation is clear.

The inheritance data with secondary trisomics presented above have increased our understanding of at least four linkage groups as follows.

The arm locations of three genes of the linkage group $l-m s-8-r e-c p t-$ $b u$ - ch- $d l$-al of chromosome 8 had previously been determined by means of radiation-induced deficiencies (Rick and Khush, 1966), $l$ allocated to 8S and $b u$ and $d l$ to $8 \mathrm{~L}$. Additionally, $c p t$ was positioned to $8 \mathrm{~L}$ by means of a telotrisomic test. The exact position of the centromere was not known, although it was understood that it must lie somewhere in the $l-c p t$ interval. Because $r e$ gave a disomic ratio with $2 \mathrm{n}+8 \mathrm{~L} \cdot 8 \mathrm{~L}$ (table 3 ) in the present studies, it must lie on $8 \mathrm{~S}$, and the centromere can now be mapped between $r e$ and $c p t$. The closeness of $r e$ to the centromere is evident from the chromosome type segregation obtained for this gene among the primary trisomic fraction of the progeny.

The two markers, $n v$ and $a h$, of the linkage group for chromosome 9 had been located to $9 \mathrm{~L}$ by means of radiation-induced deficiencies (Khush, Rick and Robinson, 1964), and marm had been located to 9L by means of a tertiary trisomic test (Khush and Rick, 1967e). To determine the arm location of pum, $2 n+9 S \cdot 9 S$ was made heterozygous for the marker group pum-ah-marm. As the data of table 3 show, all three genes gave disomic ratios among the secondary trisomic subpopulations. These results confirm the previous locations of ah and marm and limit pum to $9 \mathrm{~L}$ as well. One marker of the Verkerk series (No. 377-20aa) was found to be closely linked to $a h$ by Robinson (1966). Seeking a marker for 9S, we tested this gene with $2 n+9 L \cdot 9 L$, and, as the data of table 3 show, it gave disomic ratios and must therefore lie on 9S. The chromosomal location of this gene can be verified from the trisomic ratio obtained in the primary trisomic fraction of the progeny. Being identical in phenotype with wd-another marker of 9 which is known to be closely linked to $a h$ - this new marker is suspected to be identical or allelic with it.

In the latest chromosome map of tomato (Clayberg, Butler, Kerr, Rick and Robinson, 1966), the linkage group of chromosome 10 is shown to be $l g-p e-u-o l i-h-1-2-t-a g-X a$. The markers $t$, $a g$ and $X a$ were previously located on $10 \mathrm{~L}$ by means of a tertiary trisomic test (Khush and Rick, $1967 e$ ), but these determinations did not reveal the orientation of the linkage map or the position of the centromere. To accomplish this purpose, $l g, h$ and $u$ were tested against $2 \mathrm{n}+10 \mathrm{~L} \cdot 10 \mathrm{~L}$. As discussed already, $l g$ gave disomic segregation among the triplo- 10 fraction of the segregating progeny of $2 \mathrm{n}+10 \mathrm{~L} \cdot 10 \mathrm{~L}$ (table 3 ) and thus cannot be situated on chromosome 10 . Butler (1954) reported $l g$ and allocated it to chromosome 10 . The latest map distance between pe and $u$ was reported as $43 \mathrm{cM}$ by Clayberg, Butler, Kerr, Rick and Robinson (1966). Bona fide linkages of the latter magnitude are notoriously difficult to verify. It is therefore evident that neither pe nor $l g$ is located on chromosome 10 and that the reported linkage between $p e$ and $u$ must be spurious. On the other hand, $h$ gave trisomic ratios amongst secondary trisomic as well as primary trisomic subfamilies and must therefore 
be located on 10L. This secondary trisomic family was also segregating for $a g$, and trisomic ratios were obtained as expected for this marker. The fact that double reduction was observed for ag but not for $h$ amongst the triplo- 10 subfamily argues for a proximal position of $h$ and distal for ag on 10L. The map must therefore be oriented as indicated above, but with $u$ at the 0 position instead of $l g$. A backcross test between $u$ and $2 \mathrm{n}+10 \mathrm{~L} \cdot 10 \mathrm{~L}$ segregated disomically for $u$ in the diploid and secondary trisomic fractions of the population (table 3), hence $u$ must reside in $10 \mathrm{~S}$ and the centromere between $u$ and $h$. In the same progeny, $t$ segregated in trisomic fashion amongst $2 \mathrm{n}+10 \mathrm{~L} \cdot 10 \mathrm{~L}$ individuals as expected. The greenhouse environment of this test did not permit detection of triplo-10, whereas the phenotype of $2 \mathrm{n}+10 \mathrm{~L} \cdot 10 \mathrm{~L}$ was distinct.

Additional evidence of a distal locus for ag is provided by its segregation in the diploid fraction. The observed proportion of ag/ag (38.1 per cent.) is significantly lower than expected $\left(\chi^{2}=14 \cdot 2 * * *\right)$ for a locus close to the centromere and closely approximates the 35 per cent. expected for a distant, freely recombining locus if account is taken of the fact that 60 per cent. of the cells of this trisomic were observed to form trivalents.

Only two genes have been located on chromosome 12 to date: alb was delimited to 12S (Khush and Rick, 1966a) and $f d$ to 12L (Rick, Khush and Andrásfalvy, 1967) by means of radiation-induced deficiencies. The two arms of chromosome 12 are so similar in length and chromomere pattern that it is difficult to distinguish between them in cytological preparations. Being thus somewhat uncertain of the arm location of $f d$, we tested $f d$ as well as alb with $2 \mathrm{n}+12 \mathrm{~L} \cdot 12 \mathrm{~L}$. Since both gave disomic ratios in the secondary trisomic fractions of their progenies, they must lie on 12S. Although the primary trisomic fraction of each family was very small, the fact that a recessive homozygote appeared for alb but none for $f d$ (table 3 ) suggests that $f d$ might be proximally located to alb.

\section{TRANSMission RATES}

The data on the transmission rates of seven secondary trisomics are presented in table 4 . The progenies of the remaining two secondaries, $2 n+7 L \cdot 7 L$ and $2 n+3 S \cdot 3 S$, have not yet been grown as they were obtained only recently. For five of these trisomics, the data on transmission rates have been calculated from the data of table 3 , while for $2 n+6 L \cdot 6 L$ and $2 n+7 S \cdot 7 S$ backcross progenies with cv. VF36 as the male parent were grown. It should be noted that all the progenies from which these data were taken had high heterozygosity, which tends to enhance transmission rates of extra chromosomal types.

As may be seen from table 4, the transmission rates of secondary trisomics varied between 0.0 per cent. for $2 n+6 L \cdot 6 L$ and $33 \cdot 6$ per cent. for $2 n+7 S \cdot 7 S$. The tremendous difference between the transmission rates can be explained on the basis of differential unbalance caused by the extra isochromosomes. Extra $6 \mathrm{~L} \cdot 6 \mathrm{~L}$ causes so much unbalance that none of the $\mathrm{n}+6 \mathrm{~L} \cdot 6 \mathrm{~L}$ gametophytes function. Similarly, the viability of $\mathrm{n}+8 \mathrm{~L} \cdot 8 \mathrm{~L}$ gametophytes appears to be very low. In general, the extra isochromosomes of long arms cause greater unbalance than those of short arms.

The proportion of related primary trisomics in the progenies of tomato secondaries varies widely (table 4 ) like the classical ones of Datura stramonium. 
No primaries appeared in the progeny of $2 n+7 S \cdot 7 S$, but a maximum of 14.9 per cent. was observed in the progeny of $2 n+10 \mathrm{~L} \cdot 10 \mathrm{~L}$. The corresponding range for Datura primaries was 0.06 per cent. in the progeny of $2 n+19 \cdot 19$ to 10.01 per cent. in the progeny of $2 n+9 \cdot 9$ (Avery, Satina and Rietsema, 1959). The ratio of related primaries to the parental secondaries was also interestingly variable. In the progeny of $2 n+6 L \cdot 6 L$, for example, only primaries but no secondaries appeared; for $2 n+7 S \cdot 7 S$ the opposite was obtained. The proportion of primaries was more than double the proportion of secondaries in the progeny in $2 n+8 \mathrm{~L} \cdot 8 \mathrm{~L}$, while it was lower in the progenies of other secondaries (table 4 ). The cytological requirements which

\section{TABLE 4}

Transmission rates of secondary trisomics

\begin{tabular}{|c|c|c|c|c|c|c|c|}
\hline \multirow[b]{3}{*}{ Trisomic } & \multicolumn{7}{|c|}{ Progeny } \\
\hline & \multirow{2}{*}{ Total } & \multirow[b]{2}{*}{$2 n$} & \multicolumn{2}{|c|}{ Parental 2n+l } & \multicolumn{2}{|c|}{$\begin{array}{l}\text { Related primary } \\
\qquad 2 n+1\end{array}$} & \multirow{2}{*}{$\begin{array}{l}\text { Unrelated * } \\
\qquad 2 \mathrm{n}+1\end{array}$} \\
\hline & & & No. & $\%$ & No. & $\%$ & \\
\hline $2 n+6 L \cdot 6 L$ & 681 & 510 & 0 & $0 \cdot 0$ & 71 & $10 \cdot 4$ & 0 \\
\hline $2 n+7 S \cdot 7 S$ & 259 & 170 & 87 & $33 \cdot 6$ & 0 & $0 \cdot 0$ & 2 \\
\hline $2 n+8 L \cdot 8 L$ & 268 & 216 & 15 & $5 \cdot 6$ & 34 & $12 \cdot 7$ & 3 \\
\hline $2 n+9 S \cdot 9 S$ & 298 & 234 & 61 & $20 \cdot 4$ & 3 & $1 \cdot 0$ & 0 \\
\hline $2 n+9 L \cdot 9 L$ & 397 & 300 & 67 & $15 \cdot 3$ & 29 & $7 \cdot 3$ & 1 \\
\hline $2 \mathrm{n}+10 \mathrm{~L} \cdot 10 \mathrm{~L}$ & 681 & 447 & 130 & $19 \cdot 1$ & 102 & $14 \cdot 9$ & 2 \\
\hline $2 \mathrm{n}+12 \mathrm{~L} \cdot 12 \mathrm{~L}$ & 594 & 496 & $81 * *$ & $15 \cdot 3$ & 17 & $2 \cdot 8$ & 0 \\
\hline
\end{tabular}

give rise to $\mathrm{n}+\mathrm{l}$ gametes having a primary extra chromosome instead of a secondary were discussed in the section on cytology. The correlation between low frequency of trivalent formation in $2 n+7 S \cdot 7 S$ and $2 n+9 S \cdot 9 S$ on the one hand (table 2) and the low frequency of the primaries in their progenies on the other (table 4) is clear.

A few unrelated primary trisomics appeared among the progenies of some of the secondaries (tables 3 and 4 ). $2 n+7 S \cdot 7 S$ yielded two triplo- 4 ; $2 \mathrm{n}+8 \mathrm{~L} \cdot 8 \mathrm{~L}$, one triplo- 10 and two triplo-7; $2 \mathrm{n}+9 \mathrm{~L} \cdot 9 \mathrm{~L}$, one triplo-5; $2 \mathrm{n}+10 \mathrm{~L} \cdot 10 \mathrm{~L}$, one triplo-2 and one triplo-12. These trisomics undoubtedly appeared as a result of non-disjunction in other members of the complement. The results suggest that the presence of extra isochromosomes might increase the spontaneous rates of non-disjunction in unrelated chromosomes.

\section{Discussion}

Of the group of nine secondary trisomics of tomato reported in this paper, $2 \mathrm{n}+6 \mathrm{~L} \cdot 6 \mathrm{~L}$ is probably most interesting. This trisomic originated spontaseously in the progeny of haplo-4S-10S. The plant had good vigour, flowered profusely and set a moderate fruit load. When a large progeny was raised, the parental trisomic did not appear, only diploid and triplo-6. Clearly gametophytes that receive an extra $6 \mathrm{~L} \cdot 6 \mathrm{~L}$ isochromosome do not tolerate such unbalance. It was shown earlier (Khush and Rick, 1967b) that gametophytes carrying extra $2 \mathrm{~L} \cdot 2 \mathrm{~L}$ or the $3 \mathrm{~L} \cdot 3 \mathrm{~L}$ isochromosomes do not 
survive either. The gamete which gave rise to the $2 n+6 L \cdot 6 L$ plant was probably produced by a gametophyte carrying an extra $6 \mathrm{~L}$ telocentric, which was converted into an isochromosome either during gametogenesis or during the early ontogeny of the sporophyte. Thus $2 n+2 L \cdot 2 L$ and $2 n+3 L \cdot 3 L$ plants may also appear in this same fashion, but, like $2 n+6 L \cdot 6 L$, they will never transmit the extra isochromosome to the next generation. On the basis of arm length, the same is likely to hold for $2 \mathrm{n}+1 \mathrm{~L} \cdot 1 \mathrm{~L}$ and $2 \mathrm{n}+4 \mathrm{~L} \cdot 4 \mathrm{~L}$.

Thus all the 24 possible secondary trisomics may be viable sporophytes, but the gametophytes carrying an extra $1 \mathrm{~L} \cdot 1 \mathrm{~L}, 2 \mathrm{~L} \cdot 2 \mathrm{~L}, 3 \mathrm{~L} \cdot 3 \mathrm{~L}, 4 \mathrm{~L} \cdot 4 \mathrm{~L}$ or $6 \mathrm{~L} \cdot 6 \mathrm{~L}$ probably do not survive. Transmissible secondary trisomics for the other 19 arms should be obtainable. Ten are already known: nine reported here and one by Moens (1965).

Compared to such other genetically well-known plant species as maize, the tolerance limits for deficiencies and duplications in tomato are narrower. In maize, monosomics for four different chromosomes (Baker and Morgan, 1966), including the longest chromosome which measures $82.4 \mu$ or 14.9 per cent. of the total length of the complement, have been obtained. The longest deficiency obtained in tomato is that of chromosome 11 which measures $24.8 \mu$ or 6.4 per cent. of the total length of the complement. At the haploid level, no euchromatic deficiency, and only one small heterochromatic deficiency, have been found to survive in tomato. In maize, however, many small deficiencies of euchromatic regions are known to survive and transmit to the next generation (McClintock, 1941, 1944; Patterson, 1952). Even rather large deficiencies in maize comprising onefourth of the chromosome arm have transmitted to the next generation through the female side (Stadler, 1933, 1935).

The maximum number of extra chromosomes tolerated by the sporophytes of tomato is three (Rick and Barton, 1954), and, in these triple trisomics, only the smaller chromosomes of the complement are found to be extra. In maize, plants with six to seven extra chromosomes are known to be viable (McClintock, 1929). Thus, maize tolerates higher levels of duplication and deficiency than the tomato.

Differences in tolerance for deficiencies and duplications are usually explained in terms of levels or degrees of polyploidy. The comparison of experimental evidence available for maize and tomato therefore indicates that the latter is even more "diploid" than the former. This conclusion is also supported by observations of lowered phenotypic effect of extra chromosomes and more frequent occurrence of duplicate genes in maize than in tomatoes.

\section{Summary}

1. Nine different secondary trisomics of the tomato were investigated. Three originated from haplo-triplo-disomics, three from double iso-compensating trisomics, two from tertiary monosomics, and one arose independently on three occasions from different kinds of deficiencies. The most productive source in our experience is karyotypes that possess isochromosomes which were obtained by induced breakage and reunion of the centromere.

2. Chromosomal associations were studied at pachytene and diakinesis, and relative frequencies of various diakinetic configurations were found to 


\section{Plate I}

Photomicrographs of tomato pachytene chromosomes $\times 2000$.

Fig. 1.--Internally paired isochromosome $3 \mathrm{~S} \cdot 3 \mathrm{~S}$ in $2 \mathrm{n}+3 \mathrm{~S} \cdot 3 \mathrm{~S}$.

FIG. 2.-Trivalent association in $2 n+3 S \cdot 3 S$. Only heterochromatic part of $3 \mathrm{~L}$ is visible.

Fig. 3.-Trivalent association in $2 \mathrm{n}+6 \mathrm{~L} \cdot 6 \mathrm{~L}$. Only about the proximal half of paired $6 \mathrm{~L}$ 's can be seen.

FIG. 4.-(a) Internally paired $6 \mathrm{~L} \cdot 6 \mathrm{~L}$ isochromosome in $2 \mathrm{n}+6 \mathrm{~L} \cdot 6 \mathrm{~L}$.

(b) Interpretive drawing of a.

Fig. 5.-Trivalent association in $2 \mathrm{n}+7 \mathrm{~S} \cdot 7 \mathrm{~S}$.

Frg. 6.-Trivalent association in $2 \mathrm{n}+8 \mathrm{~L} \cdot 8 \mathrm{~L}$. One chiasma can be easily seen in the $8 \mathrm{~L}$ 's.

Fig. 7.-Internally paired isochromosome $8 \mathrm{~L} \cdot 8 \mathrm{~L}$ in $2 \mathrm{n}+8 \mathrm{~L} \cdot 8 \mathrm{~L}$.

Frg. 8.-Internally paired isochromosome $7 \mathrm{~S} \cdot 7 \mathrm{~S}$ in $2 \mathrm{n}+7 \mathrm{~S} \cdot 7 \mathrm{~S}$.

Fig. 9.-Internally paired isochromosome $9 S \cdot 9 S$ in $2 n+9 S \cdot 9 S$.

Frg. 10.-Trivalent association in $2 n+9 S \cdot 9 S$.

Frg. 11.-Trivalent association in $2 n+9 L \cdot 9 L$. One chiasma can be easily seen in the 9L's.

Fig. 12.-Internally paired isochromosome $9 \mathrm{~L} \cdot 9 \mathrm{~L}$ in $2 \mathrm{n}+9 \mathrm{~L} \cdot 9 \mathrm{~L}$.

Frg. 13.--Trivalent association in $2 \mathrm{n}+10 \mathrm{~L} \cdot 10 \mathrm{~L}$. Two chiasmata can be seen in the 10L's.

Frg. 14.- Internally paired isochromosome $10 \mathrm{~L} \cdot 10 \mathrm{~L}$ in $2 \mathrm{n}+10 \mathrm{~L} \cdot 10 \mathrm{~L}$.

Frg. 15.-Internally paired isochromosome $12 \mathrm{~L} \cdot 12 \mathrm{~L}$ in $2 \mathrm{n}+12 \mathrm{~L} \cdot 12 \mathrm{~L}$.

Frg. 16. - Trivalent association in $2 \mathrm{n}+12 \mathrm{~L} \cdot 12 \mathrm{~L}$. One chiasma can be seen in the 12L's. 


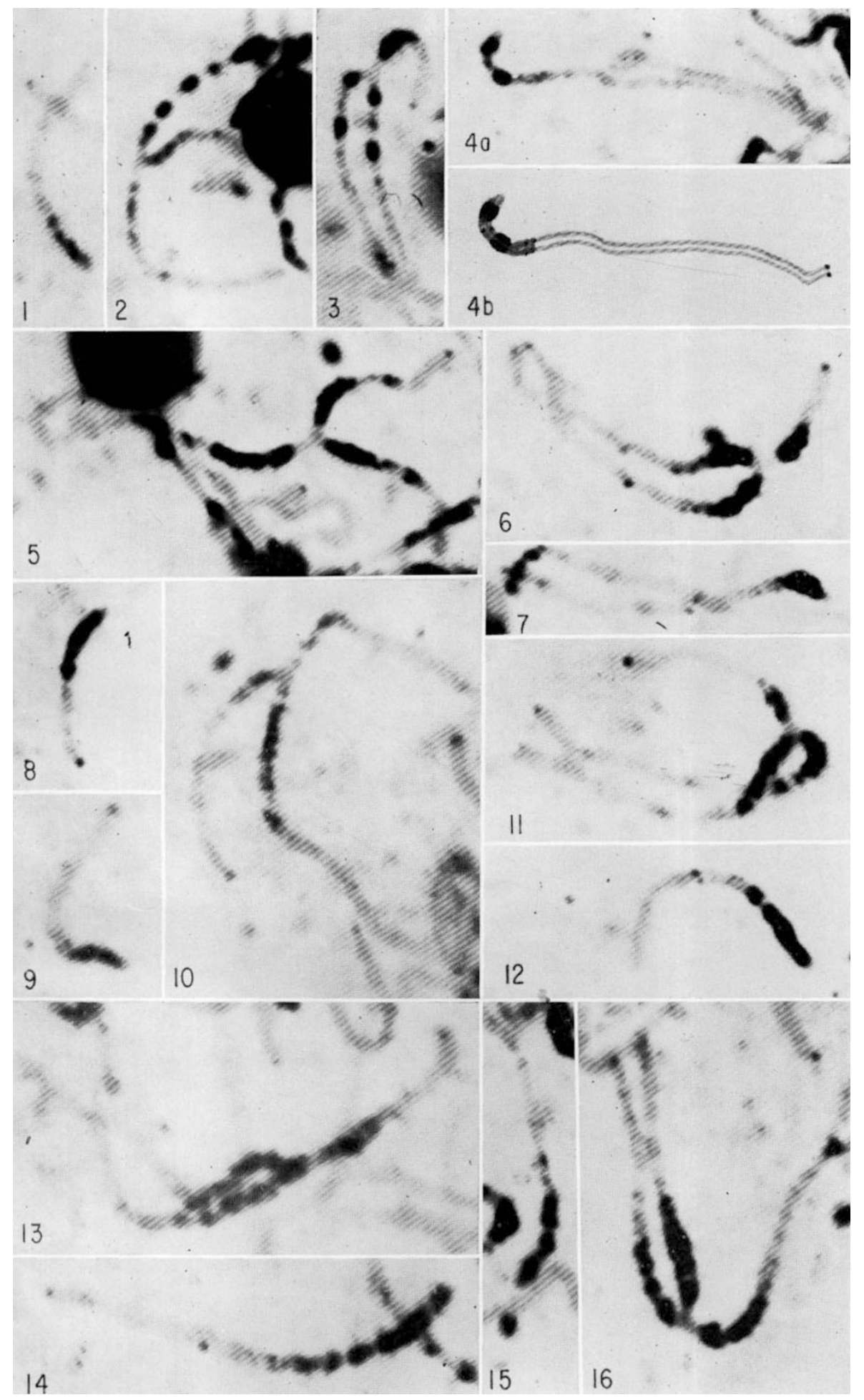



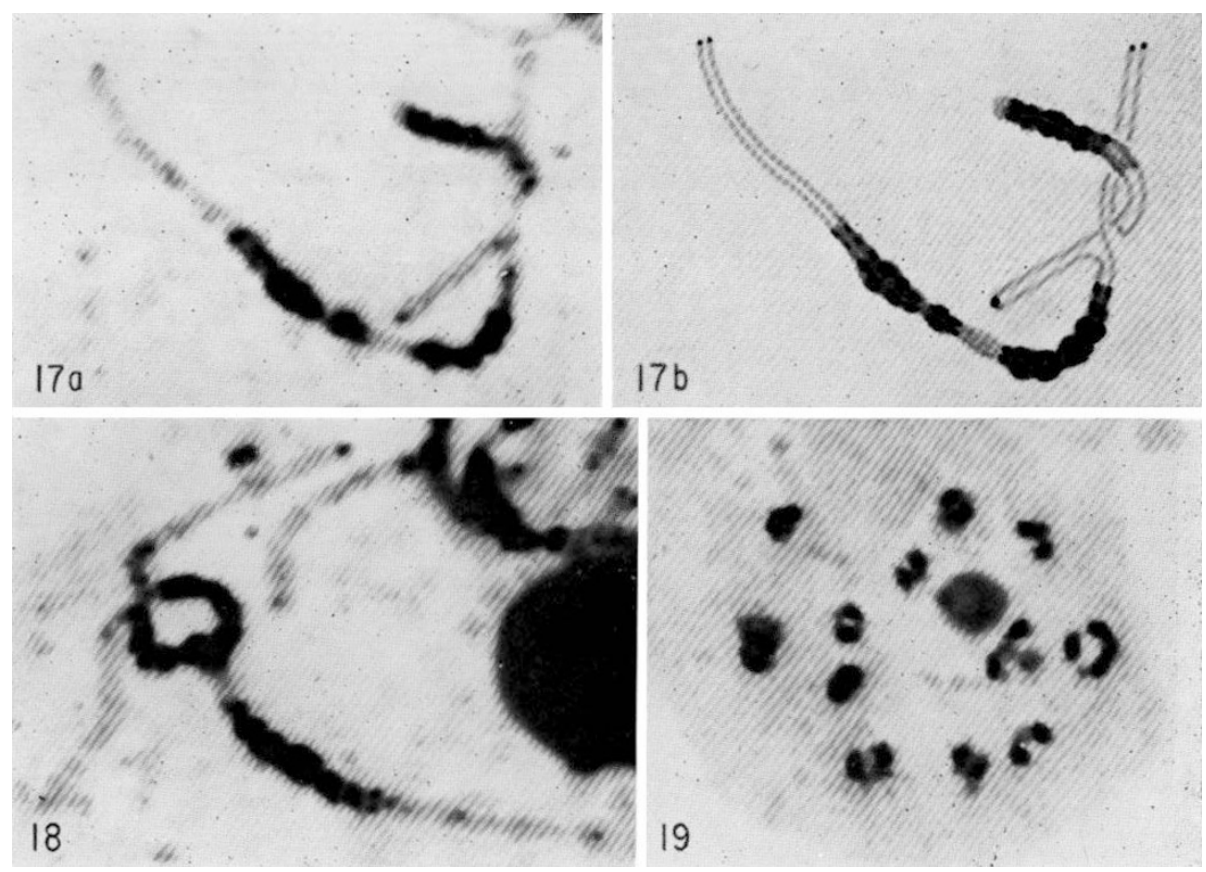

Photomicrographs of tomato chromosomes. Figs. 17 and 18 at pachytene stage of meiosis $\times 2000$; fig. 19 at diakinesis $\times 600$.

FIG. 17.-(a) Trivalent association in $2 \mathrm{n}+12 \mathrm{~L} \cdot 12 \mathrm{~L}$. (b) Interpretive drawing of $a$.

Fig. 18.- Trivalent association in $2 \mathrm{n}+12 \mathrm{~L} \cdot 12 \mathrm{~L}$. One chiasma can be seen in the 12L's.

FIG. 19. - 11 bivalents and 1 ring trivalent in $2 \mathrm{n}+10 \mathrm{~L} \cdot 10 \mathrm{~L}$. 
agree roughly with expectations based on arm lengths and number of chiasmata. When univalent, the isochromosomes tended to pair internally, suffer chiasmata, and form ringlets. The low frequencies of trivalent formation in $2 n+7 S \cdot 7 S$ and $2 n+9 S \cdot 9 S$ led to the low yield of corresponding primary trisomics in their progeny.

3. The transmission rates of isochromosomes varied greatly, none having been recovered from $2 n+6 L \cdot 6 L$ and few from $2 n+8 L \cdot 8 L$, presumably because such imbalance is at the limit of gametophytic tolerance in tomatoes. For the same reason no haplo-triplo-disomic progeny were yielded.

4. Comparisons of gross morphology between secondaries and their corresponding primaries revealed that the effect wrought by long arms is disproportionate to their relative length while that by short arms is generally unpredictable. The two arms may exert an opposing influence on the same character, in which case the effect of the long arm always dominates that of the short one.

5. The first inheritance data for monogenic segregation in secondary trisomics of any organism are presented. For those secondaries that yield corresponding primaries in their progeny, such segregations can identify the chromosome on which the gene in question is located, the arm and the relative distance from the centromere. Information was thereby obtained for four tomato chromosomes to clarify the relationships between chromosomes and their genetic linkage maps. For chromosomes 8, 9 and 10 the centromere positions could be placed more precisely on the maps, and for chromosome 10 a previously reported linkage was proven to be spurious.

Acknowledgment.-Research was supported in part by grant G.M. 06209 of the National Institutes of Health, U.S.P.H.S.

\section{References}

Avery, A. G., satina, s., And RIEtsema, J. 1959. Blakeslee: The Genus Datura. 289 pp. The Ronald Press Company, New York.

BAKER, R. L., AND MORGAN, D. T., JR. 1966. Monosomics in maize induced by X-irradiation of the pollen. Cytologia, 31, 172-175.

BELLING, J., AND BLAKESLEE, A. F. 1924. The configurations and sizes of the chromosomes in the trivalents of 25 chromosome Daturas. Proc. Nat. Acad. Sci. (U.S.A.), 10, 116-120.

BLAKESLEE, A. F. 1924. Distinction between primary and secondary chromosomal mutants in Datura. Proc. Nat. Acad. Sci. (U.S.A.), 10, 109-116.

BLAKESLEE, A. F., AND AVERY, A.G. 1938. Fifteen-year breeding records on $2 n+1$ types in Datura stramonium. Cooperation in Research, Carnegie Inst. Washington Publ., 501, 315-351. Butler, L. 1954. Light green foliage, lg. Rept. Tomato Genetics Coop., 4, 9.

CLAYBERG, C. D., BUTLER, L., KERR, E. A., RICK, C. M., AND ROBINSON, R. W. 1966. Third list of known genes in the tomato. Four. Hered., 57, 188-196.

KHUSH, G. S., AND RICK, C. M. 1966a. The origin, identification and cytogenetic behavior of tomato monosomics. Chromosoma, 18, 407-420.

KHUSH, G. s., AND RICK, c. M. 1966b. The use of tertiary trisomics in linkage mapping. Genetics, $54,343$.

KHUSH, G. S., AND RiCK, C. M. 1967a. Novel compensating trisomics of the tomato: cytogenetics, monosomic anaylsis, and other applications. Genetics, 55, 297-307.

KHUSH, G. S., AND RICK, c. M. 1967b. Haplo-triplo-disomics of the tomato: origin, cytogenetics, and utilization as a source of secondary trisomics. Biol. Zbl., 86, (in press).

KHUSH, G. s., AND RICK, c. M. 1967c. Studies on the linkage map of chromosome 4 of the tomato and on the transmission of induced deficiencies. Genetica, 38, 74-94.

KHUSH, G. S., AND RICK, C. M. 1967d. Tomato telotrisomics: origin, identification, and use in linkage mapping. Cytologia, 32, (in press). 
KHUSH, G. s., AND Rick, с. M. 1967e. Tomato tertiary trisomics: origin, identification, morphology and use in determining position of centromeres and arm location of markers. Canad. 7. Genet. Cytol., 9, 610-631.

KHUSH, G. s., RICK, C. M., AND ROBINSON, R. w. 1964. Genetic activity in a heterochromatic chromosome segment of the tomato. Science, 145, 1432-1434.

MCcLintock, B. 1929. A cytological and genetical study of triploid maize. Genetics, 14, $180-222$.

MCCLINTOCK, B. 1941. The association of mutants with homozygous deficiencies in Zea mays. Genetics, 26, 542-571.

MCCLiNTOCK, в. 1944. The relation of homozygous deficiencies to mutations and allelic series in maize. Genetics, 29, 478-502.

MOENS, P. 1965. The transmission of a heterochromatic isochromosome in Lycopersicon esculentum. Canad. 7. Genet. Cytol., 7, 296-303.

PATtERSON, E. B. 1952. The use of functional deficient-duplicate gametes in locating genes in maize. Genetics, 37, 612 .

Rhoades, м. м. 1933. A secondary trisomic in maize. Proc. Nat. Acad. Sci. (U.S.A.), 19, 1031-1038.

RHOADES, M. M. 1940. Studies of a telocentric chromosome in maize, with reference to the stability of its centromere. Genetics, 25, 483-520.

RIGK, c. M., AND BARTON, D. w. 1954. Cytological and genetical identification of the primary trisomics of the tomato. Genetics, 39, 640-666.

RICK, C. M., AND KHUSH, G.s. 1966. Chromosome engineering in Lycopersicon. In Chromosome Manipulations and Plant Genetics. (Ed. R. Riley and K. R. Lewis) a supplement to Heredity, 20, 8-20.

RICK, c. M., AND KHUSH, G. s. 1968. Cytogenetic explorations in the tomato genome. In Seminars in Genetics (Ed. R. Bogart). Corvallis, Oregon.

RICK, C. M., KHUSH, G. S., AND ANDRÁsfalvY, A. 1967. Flecked dwarf, a marker for 12L. Rept. Tomato Genetics Coop., 17, 45-46.

Robinson, R. w. 1966. In: New Mutant Program. Rept. Tomato Genetics Coop., 16, 3-4.

sears, E. R. 1954. The aneuploids of common wheat. Missouri Agr. Exp. Sta. Res. Bull., 572.

SEN, N. K. 1952. Isochromosomes in tomato. Genetics, 37, 227-241 .

STADLER, L. J. 1933. On the genetic nature of induced mutations in plants. II. A haploviable deficiency in maize. Missouri Agr. Exp. Sta. Res. Bull., 204, 1-29.

STADLER, L. J. 1935. Genetic behavior of a haplo-viable internal deficiency in maize. Amer. Nat., 69, 80-81. 